\title{
A REVIEW OF MEDICATION RELATED INFORMATION IN PAEDIATRIC GASTROENTEROLOGY OUT-PATIENT CLINIC LETTERS
}

Monisha Sahni,, Jeff Aston, ${ }^{2}$ David Terry ${ }^{1,2}$. 'Aston University; ${ }^{2}$ Birmingham Children's Hospital NHS Foundation Trust

10.1136/archdischild-2015-308634.43

Aims The aim of this study was to evaluate the accuracy and completeness of medication related information provided in out-patient clinic letters.

Method Single-site, cross-sectional, retrospective study. The medication related information included in 50 gastroenterology out-patient clinic letters was reviewed. The evaluation criteria used was adapted from the Sheffield Assessment Instrument for Letters. ${ }^{1} 2$ Clinic letters were assessed against five key themes: patient details, diagnosis/current medical issues, medication name(s), completeness of medication details and changes to the patient's care plan. Each letter was scored against these five criteria, where each criterion was awarded a value between 0 and 2. Therefore each clinic letter was scored out of ten. A perfect score of ten indicated no errors or omissions. The information provided in the clinic letters was reconciled with the corresponding out-patient prescription. Data were analysed using MS-Excel 2010 to obtain descriptive statistics.

Results 46 (92\%) out-patient letters were associated with at least one error/omission. In total, 94 errors/omissions were identified. The most common omission was a lack of allergy/sensitivity information in 46/50 (92\%) cases. Medication related information included 41/94 (43.6\%) errors/omissions. These included a lack of formulation $(26 / 41,63.4 \%)$, omission of strength/concentration $(8 / 41,19.5 \%)$ and a lack of dose regimen (4/41, 9.8\%). Errors/omissions were identified in patient details including a missing weight in 5/50 (10\%) cases. No errors/omissions were identified in the details of diagnosis/current medical conditions.

The mean score achieved was 8.2 (range 6-10). Four clinic letters achieved a score of 10 (error/omission free).

Conclusion Out-patient clinic letters are a way of communicating information about patients' medicines. This study demonstrates that these letters contain omissions/errors that may be 
important when patient care is shared or managed across healthcare sectors. It is known that when patients move between care providers the risk of miscommunication and unintended changes to medicines remains a significant problem. ${ }^{3}$ The most common medication omissions/errors (allergy status, drug formulation, strength, dosage regimen) might be reduced by involving pharmacists in communicating medication related information or using a standardised clinic letter designed to facilitate a complete record of medication use. Further research is needed to determine the extent of inaccurate discharge letters in other specialties and how this form of communication may be optimised.

\section{REFERENCES}

1 Crossley GM, Howe A, Newble D, et al. Sheffield Assessment Instrument for Letters (SAIL): performance assessment using outpatient letters. Med Educ 2001;35:1115-24

2 Chindamai P, Bush J, Gupta R et al. Evaluation of Outpatient Clinic Letters for Medication Errors. Eur J Hosp Pharm 2014;21:A14

3 Royal Pharmaceutical Society. Keeping patients safe when they transfer between care providers -getting the medicines right. http://www.rpharms.com/ current-campaigns-pdfs/rps-transfer-of-care-final-report.pdf (accessed 2 Jul 2014). 